\title{
Estado Nutricional de Pacientes Pediátricos Recém-Diagnosticados com Leucemia Linfoblástica Aguda em um Instituto de Referência em Oncologia do Rio de Janeiro
}

doi: https://doi.org/10.32635/2176-9745.RBC.2018v64n3.35

Nutritional Status of Pediatric Patients Newly Diagnosed with Acute Lymphoblastic Leukemia at an Institute of Reference

in Oncology of Rio de Janeiro

Estado Nutricional de Pacientes Pediátricos Recién Diagnosticados con Leucemia Linfoblástica Aguda en un

Instituto de Referencia en Oncología de Río de Janeiro

Karina Andrade Moreira'; Ana Lucia Miranda de Carvalho²; Marcia Trindade Schramm³; Renata Brum Martucci; Leonardo Borges Murad $^{5}$; Danúbia da Cunha Antunes Saraiva ${ }^{6}$

Resumo

Introduçáo: A leucemia linfoblástica aguda (LLA) constitui a neoplasia mais comum em pediatria e a avaliação nutricional é um instrumento essencial para conhecer as condiçôes de saúde dos pacientes. O presente estudo teve como objetivo avaliar e descrever o estado nutricional de pacientes pediátricos recém-diagnosticados com LLA e verificar a relação do índice de massa corporal para idade (IMC/I) com as demais medidas antropométricas. Método: Estudo observacional, transversal, realizado com pacientes de 1 a 18 anos, recém-diagnosticados com LLA, no período de janeiro/2004 a dezembro/2009. Os dados coletados foram idade, peso corporal, estatura, prega cutânea tricipital (PCT), circunferência do braço (CB) e circunferência muscular do braço (CMB). O IMC foi calculado e a classificação IMC/I utilizada na análise. O teste Qui-quadrado de Pearson e Exato de Fisher foram utilizados para analisar a associaçáo entre os parâmetros antropométricos. Resultados: Foram incluídos no estudo 54 pacientes. A classificaçáo do "estado nutricional adequado" foi a mais frequente em todos os parâmetros antropométricos. Em adição, observou-se que a associaçáo do estado nutricional pela CMB com o IMC/I ( $\mathrm{p}=0,001)$ demonstra que este último pode ser adequado para classificaçáo dos pacientes pediátricos com LLA ao diagnóstico. E a concordância da $\mathrm{CB}$ com a CMB $(\mathrm{p}=0,01)$ de $43 \%(\mathrm{p}=0,001)$ também ratifica a utilização da $\mathrm{CB}$ frente à $\mathrm{CMB}$, por ser uma medida mais simples. Conclusáo: Na população estudada, o estado nutricional encontrava-se preservado. Assim como a associação da CMB com o IMC/I, a CB demonstrou ser um parâmetro sensível para classificar eutrofia.

Palavras-chave: Criança; Adolescente; Estado Nutricional; Avaliação Nutricional; Leucemia-Linfoma Linfoblástico de Células Precursoras.

\begin{abstract}
Introduction: Acute lymphoblastic leukemia (ALL) is the most common neoplasm in pediatrics, and nutritional assessment is an essential tool for understanding patients' health conditions. The present study aimed to evaluate and describe the nutritional status of pediatric patients newly diagnosed with ALL and to verify the relation between body mass index for age (BMI-forAge) and other anthropometric measurements. Method: A cross-sectional, observational study was performed with patients from 1 to 18 years of age, newly diagnosed with ALL, from January 2004 to December 2009. The data collected were age, body weight, height, triceps skinfold (TS), arm circumference (AC) and arm muscle circumference (AMC). The BMI was calculated and the BMI-for-Age classification used in the analysis. Pearson's Chi-square test and Fisher's exact test were used to analyze the association between anthropometric parameters. Results: 54 patients were included in the study. The classification of "adequate nutritional status" was the most frequent in all anthropometric parameters. In addition, we observed that the association of nutritional status by AMC with BMI-for-Age ( $\mathrm{p}=0.001)$ shows that the BMI-for-Age may be adequate for classification of pediatric patients with ALL at diagnosis. In addition, $A C$ agreement with AMC $(\mathrm{p}=0.01)$ of $43 \%(\mathrm{p}=0.001)$ also confirmed the use of AC versus AMC, as it is a simpler measure. Conclusion: In the studied population the nutritional status was preserved. Just as the association of AMC with BMI-for-Age, AC has been shown to be a sensitive parameter for classifying eutrophy.

Key words: Child; Adolescent; Nutritional Status; Nutrition Assessment; Precursor Cell Lymphoblastic Leukemia-Lymphoma.
\end{abstract}

Resumen

Introducción: La leucemia linfoblástica aguda (LLA) constituye la neoplasia más común en pediatría y la evaluación nutricional es un instrumento esencial para conocer las condiciones de salud de los pacientes. El presente estudio tuvo como objetivo evaluar y describir el estado nutricional de pacientes pediátricos recién diagnosticados con LLA y verificar la relación del Índice de masa corporal por edad (IMC/I) con las demás medidas antropométricas. Método: Estudio observacional, transversal, realizado con pacientes de 1 a 18 años recién diagnosticados con LLA en el período de enero / 2004 a diciembre / 2009. Los datos recogidos fueron edad, peso corporal, estatura, pliegue cutáneo tricipital (PCT), circunferencia del brazo (CB) y circunferencia muscular del brazo (CMB). El IMC fue calculado y la clasificación IMC/I utilizada en el análisis. La prueba Chi-cuadrado de Pearson y Exacto de Fisher se utilizaron para analizar la asociación entre los parámetros antropométricos. Resultados: Se incluyeron en el estudio 54 pacientes. La clasificación del "estado nutricional adecuado", fue la más frecuente, en todos los parámetros antropométricos. En adición, se observo que la asociación del estado nutricional por la CMB con el IMC/I $(\mathrm{p}=0,001)$ demuestra que este último puede ser adecuado para clasificación de los pacientes pediátricos con LLA al diagnóstico. Y la concordancia de la CB con la CMB ( $\mathrm{p}=0,01)$ del 43\% ( $\mathrm{p}=0,001)$, también ratifica la utilización de la $\mathrm{CB}$ frente a la $\mathrm{CMB}$, por ser una medida más simple. Conclusión: En la población estudiada el estado nutricional se encontraba preservado. Así como la asociación de la CMB con el IMC/I, la CB demostró ser un parámetro sensible para clasificar eutrofia.

Palabras clave: Niño; Adolescente; Estado Nutricional; Evaluación Nutricional; Leucemia-Linfoma Linfoblástico de Células Precursoras.

\footnotetext{
${ }^{1}$ Instituto Nacional de Câncer José Alencar Gomes da Silva (INCA). Rio de Janeiro (RJ), Brasil. Orcid iD: https://orcid.org/0000-0001-9564-5319

${ }^{2}$ INCA. Rio de Janeiro (RJ), Brasil. Orcid iD: https://orcid.org/0000-0002-6597-1102

${ }^{3}$ INCA. Rio de Janeiro (RJ), Brasil. Orcid iD: https://orcid.org/0000-0002-3924-0517

${ }^{4}$ INCA. Rio de Janeiro (RJ), Brasil. Orcid iD: https://orcid.org/0000-0002-3354-4229

${ }^{5}$ INCA. Rio de Janeiro (RJ), Brasil. Orcid iD: https://orcid.org/0000-0001-8210-3000

${ }^{6}$ INCA. Rio de Janeiro (RJ), Brasil. Orcid iD: https://orcid.org/0000-0003-1849-2894

Endereço para correspondência: Karina Andrade Moreira. Rua Pinheiro Machado, 61, apto. 402 - Laranjeiras. Rio de Janeiro (RJ), Brasil. CEP 22231-090. E-mail: kam.nut@gmail.com.
} 


\section{INTRODUÇÃO}

Tem sido relatada na literatura uma alta prevalência de desnutriçáo no grupo oncopediátrico desde o momento do diagnóstico ${ }^{1,2}$, principalmente em países de baixa e média rendas ${ }^{3}$, fato que pode estar relacionado a questóes socioeconômicas e a efeitos metabólicos do próprio câncer.

Porém, pacientes pediátricos com leucemia parecem ter menor comprometimento do estado nutricional ao diagnóstico do que pacientes com tumores sólidos ${ }^{1,4}$. Crianças com leucemia linfoblástica aguda (LLA), neoplasia mais comum em pediatria, apresentam geralmente um estado nutricional preservado, o que está associado à menor morbidade e mortalidade do que aqueles que apresentam excesso de peso e obesidade ${ }^{5}$, assim como desnutrição ${ }^{6}$.

A avaliação nutricional é uma abordagem abrangente que visa a definir o estado nutricional por meio do histórico clínico, nutricional, exame físico, medidas antropométricas e exames laboratoriais ${ }^{7}$. A Sociedade Brasileira de Pediatria reforça que a avaliação nutricional é um instrumento essencial para que os profissionais da área conheçam as condiçóes de saúde dos pacientes pediátricos e que levem em conta a anamnese alimentar, assim como o exame físico e a avaliação da composição corporal (antropometria) e os exames laboratoriais8.

O diagnóstico nutricional baseado apenas no índice de massa corporal para idade (IMC/I) e nas medidas de peso corporal e estatura pode náo fornecer informaçóes precisas do estado nutricional ${ }^{9}$. Tendo em vista o impacto que o estado nutricional possui sobre o prognóstico e tratamento ${ }^{10}$, associar outras medidas antropométricas como prega cutânea tricipital (PCT), circunferência do braço $(\mathrm{CB})$ e circunferência muscular do braço $(\mathrm{CMB})$, que são medidas de fácil aplicabilidade na prática clínica e que visam a avaliar a composição corporal, contribui para determinar um diagnóstico nutricional mais preciso ${ }^{11-13}$.

Portanto, considerando a importância de conhecer o estado nutricional de pacientes pediátricos com neoplasias, o presente estudo teve como objetivo avaliar e descrever o estado nutricional de crianças e adolescentes recém-diagnosticados com LLA e verificar a relação do IMC/I com as demais medidas antropométricas em uma coorte hospitalar em um Instituto de referência em oncologia do Rio do Janeiro.

\section{MÉTODO}

Estudo observacional, transversal, que faz parte de uma coorte retrospectiva anterior ${ }^{14}$. $\mathrm{O}$ presente estudo foi descrito segundo o checklist Strobe ${ }^{15}$. Um total de
158 pacientes pediátricos entre 1 a 18 anos de idade foram matriculados com LLA na Seção de Hematologia do Hospital do Câncer I, no Instituto Nacional de Câncer José Alencar Gomes da Silva (INCA), Rio de Janeiro, Brasil, no período de janeiro de 2004 a dezembro de 2009. Destes, foram elegíveis 54 pacientes, após aplicação dos critérios de elegibilidade: diagnósticos de LLA confirmados, iniciado o tratamento no INCA, suficiência de dados nos prontuários, e Síndrome de Down (Figura 1). Muitos pacientes foram excluídos em razão da abertura de matrícula apenas para realização de radioterapia ou por terem iniciado tratamento em outras instituições de saúde do Rio de Janeiro, não sendo possível verificar a antropometria ao diagnóstico $(n=90$; 62\%). Este estudo foi aprovado pelo Comitê de Ética em Pesquisa do INCA, sob o número de protocolo CAAE 43882115.9.0000.5274.

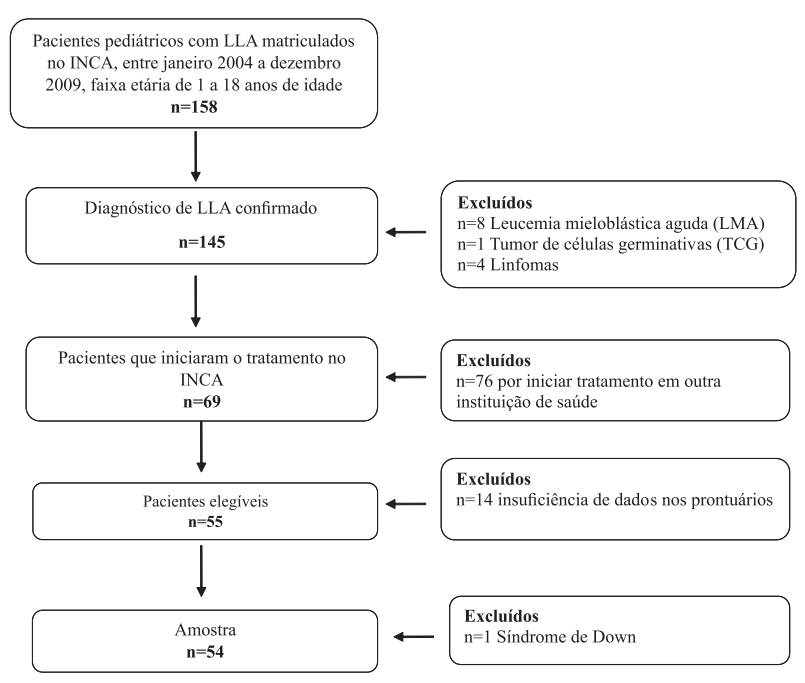

Figura 1. Fluxograma para obtenção da amostra para coleta de dados

Os dados referentes à idade (em anos e meses), peso corporal, estatura, PCT, CB e CMB dos pacientes foram coletados do prontuário. Todas as medidas antropométricas utilizadas no presente estudo foram realizadas por nutricionistas da instituição, previamente treinados.

O peso corporal e a estatura ou comprimento foram avaliados pelo indicador do IMC/I das crianças (abaixo de 5 anos) e pelo Software Anthro da Organização Mundial da Saúde (versão 3.2.2, OMS) e as crianças e adolescentes maiores de 5 anos pelo Anthro Plus (versão 1.0.4). O estado nutricional foi classificado de acordo com os parâmetros da $\mathrm{OMS}^{16,17}$ e categorizado em três grupos ${ }^{18}$ : abaixo do adequado - pacientes com z-escore $>-1$ desvio-padrão (DP) da média do IMC para idade [risco nutricional, 
magreza e magreza acentuada]; adequado - pacientes entre -1 DP e +1 DP da média do IMC/I; e acima do adequado - pacientes $>+1$ DP da média do IMC/I [risco de sobrepeso, sobrepeso, obesidade, obesidade grave].

Os parâmetros de avaliação da composição corporal foram PCT, CB e CMB. Os valores de CB, PCT e CMB foram classificados de acordo com Frisancho ${ }^{19} \mathrm{e}$ categorizados em três grupos: abaixo do adequado - $\leq$ percentil 5; adequado - entre o percentil 5 e 95; e acima do adequado - $\geq$ percentil 95 .

Foram realizadas análises descritivas de idade ao diagnóstico, sexo, estado nutricional de acordo com o parâmetro antropométrico, com suas médias, medianas e frequências, conforme o dado apresentado. As variáveis contínuas foram expressas em média e DP ou mediana com seu mínimo e máximo, conforme apropriado após realizado o teste de normalidade KolmogorovSmirnov e as categóricas apresentadas por distribuição de frequência percentual. O teste Qui-quadrado de Pearson foi utilizado nas análises de associação em tabelas de contingência $2 \times 3$ e o Teste exato de Fisher nas comparações de variáveis categóricas em tabelas de contingência $2 \times 2$. A hipótese nula era baseada na ausência de associação entre o diagnóstico nutricional de acordo com cada parâmetro antropométrico. A estatística Kappa foi realizada para verificar a concordância entre dois conjuntos de dados.

Os resultados foram considerados estatisticamente significativos quando $\mathrm{p}<0,05$ e intervalos com 95\% de confiança. O programa Statistical Package for Social Sciences (SPSS), versão 22, foi utilizado na análise dos dados.

\section{RESULTADOS}

Da população de estudo, a mediana de idade dos 54 pacientes foi 7,33 anos (1,83-17,08), sendo a maioria do sexo masculino $(55,6 \%)$. Em relação ao estado nutricional ao diagnóstico avaliado pelo IMC/I, a média do z-escore foi de $0,13(\mathrm{DP} \pm 1,19)$. As medidas de $\mathrm{CB}, \mathrm{PCT}$ e $\mathrm{CMB}$ foram realizadas em 38 pacientes e também classificadas em diferentes categorias do estado nutricional (Tabela 1). De acordo com os parâmetros nutricionais, foi verificada uma maior frequência de estado nutricional adequado em todos os parâmetros antropométricos realizados.

Com relação ao estado nutricional da coorte, observou-se que 31 pacientes $(57,4 \%)$ ao diagnóstico estavam classificados como adequado segundo o IMC/I, enquanto 23 pacientes $(42,6 \%)$ encontravam-se fora do intervalo de adequação. Para os demais parâmetros antropométricos, $\mathrm{CB}, \mathrm{PCT}$ e CMB, foram encontrados, respectivamente, 29 (76,3\%), $27(71,1 \%)$ e $35(92,1 \%)$ pacientes dentro da classificação de adequação.
Tabela 1. Estado nutricional dos pacientes pediátricos recém-diagnosticados com LLA $(n=54)$

\begin{tabular}{l|c}
\hline $\begin{array}{c}\text { Classificação de acordo com os } \\
\text { diferentes parâmetros }\end{array}$ & $\mathbf{n}(\%)$ \\
\hline $\mathrm{IMC} / \mathrm{I}$ & $11(20,4)$ \\
\hline$<$ adequado & $31(57,4)$ \\
\hline adequado & $12(22,2)$ \\
\hline$>$ adequado & \\
\hline $\mathrm{CB}^{\mathrm{a}}$ & $9(23,7)$ \\
\hline$<$ adequado & $29(76,3)$ \\
\hline adequado & $0(0)$ \\
\hline$>$ adequado & $11(28,9)$ \\
\hline PCT & $27(71,1)$ \\
\hline$<$ adequado & $0(0)$ \\
\hline adequado & \\
\hline$>$ adequado & $3(7,9)$ \\
\hline $\mathrm{CMB}^{\mathrm{a}}$ & $35(92,1)$ \\
\hline$<$ adequado & $0(0)$ \\
\hline adequado & \\
\hline$>$ adequado & \\
\hline
\end{tabular}

Nota: ${ }^{\mathrm{a}} \mathrm{n}=38$ pacientes tiveram as medidas realizadas.

Legendas: IMC/I: índice de massa corporal para idade; CB: circunferência do braço; PCT: prega cutânea tricipital; CMB: circunferência muscular do braço.

Houve associação do estado nutricional da $\mathrm{CMB}$ e do IMC/I ao diagnóstico ( $\mathrm{p}=0,001)$. É importante destacar que essa associaçáo demonstra que tanto a $\mathrm{CMB}$ como o IMC/I foram capazes de classificar a eutrofia dos pacientes pediátricos com LLA ao diagnóstico (Tabela 2). Sobre a concordância substancial entre as medidas da CB, PCT e $\mathrm{CMB}$ com o IMC/I, todas foram fracas. Observa-se que, apesar de existir uma associação do estado nutricional medido pela $\mathrm{CMB}$ e pelo IMC/I, a concordância dos resultados foi fraca (20\%), conforme pode ser observado na Tabela 3.

A Tabela 4 demonstra a associaçáo da $\mathrm{CB}$ com a $\mathrm{CMB}$ $(\mathrm{p}=0,01)$ e a concordância moderada de $43 \%(\mathrm{p}=0,001)$. É possível observar que nenhum paciente classificado como "adequado" pela CB foi classificado como "abaixo do adequado" pela CMB. Assim como a associação da CMB com o IMC/I, a CB foi sensível para o diagnóstico da eutrofia e com uma concordância prática para utilização na assistência clínica.

É importante descartar a baixa concordância da classificação do estado nutricional pela PCT em comparaçáo ao IMC/I (4\%) e à CMB (2\%). A medida de PCT requer muito treinamento, os pacientes devem estar em posição adequada para realização da medida; pois, caso contrário, pode influenciar no resultado.

\section{DISCUSSÃO}

O estado nutricional de crianças e adolescentes com câncer é primordial, uma vez que um estado nutricional 
Tabela 2. Associação do estado nutricional de acordo com diferentes parâmetros antropométricos de pacientes pediátricos ao diagnóstico de LLA $(n=38)$

\begin{tabular}{|c|c|c|c|c|}
\hline \multirow{2}{*}{$\begin{array}{c}\text { Parâmetros } \\
\text { antropométricos }\end{array}$} & \multicolumn{3}{|c|}{ Classificação do IMC/I } & \multirow{2}{*}{ p-valor } \\
\hline & < adequado & adequado & > adequado & \\
\hline \multicolumn{5}{|l|}{ CB } \\
\hline$<$ adequado & 5 & 3 & 1 & \multirow{2}{*}{0,074} \\
\hline adequado & 5 & 17 & 7 & \\
\hline \multicolumn{5}{|l|}{ PCT } \\
\hline$<$ adequado & 4 & 6 & 1 & \multirow{2}{*}{0,43} \\
\hline adequado & 6 & 14 & 7 & \\
\hline \multicolumn{5}{|l|}{ CMB } \\
\hline$<$ adequado & 3 & 0 & 0 & \multirow{2}{*}{0,01} \\
\hline adequado & 7 & 20 & 8 & \\
\hline
\end{tabular}

Nota: ${ }^{\text {a }}$ Teste Qui-quadrado de Pearson.

Legendas: IMC/I: índice de massa corporal para idade; CB: circunferência do braço; PCT: prega cutânea tricipital; CMB: circunferência muscular do braço.

Tabela 3. Concordância do estado nutricional de acordo com diferentes parâmetros antropométricos de pacientes pediátricos ao diagnóstico de LLA $(n=38)$

\begin{tabular}{|c|c|c|c|c|c|}
\hline \multirow{2}{*}{$\begin{array}{c}\text { Parâmetros } \\
\text { antropométricos }\end{array}$} & \multicolumn{3}{|c|}{ Classificação do IMC/I } & \multirow{2}{*}{\multicolumn{2}{|c|}{ Kappa/p-valor }} \\
\hline & < adequado & adequado & $>$ adequado & & \\
\hline \multicolumn{6}{|l|}{ CB } \\
\hline$<$ adequado & 5 & 3 & 1 & \multirow{2}{*}{0,21} & \multirow[b]{2}{*}{0,05} \\
\hline adequado & 5 & 17 & 7 & & \\
\hline \multicolumn{6}{|l|}{ PCT } \\
\hline$<$ adequado & 4 & 6 & 1 & \multirow{2}{*}{0,04} & \multirow{2}{*}{0,71} \\
\hline adequado & 6 & 14 & 7 & & \\
\hline \multicolumn{6}{|l|}{ CMB } \\
\hline$<$ adequado & 3 & 0 & 0 & \multirow{2}{*}{0,20} & \multirow{2}{*}{0,07} \\
\hline adequado & 7 & 20 & 8 & & \\
\hline
\end{tabular}

Legendas: IMC/I: índice de massa corporal para idade; CB: circunferência do braço; PCT: prega cutânea tricipital; CMB: circunferência muscular do braço.

Tabela 4. Associação e concordância da CMB para determinação da adequação nutricional com os demais parâmetros de avaliação da composição corporal $(n=38)$

\begin{tabular}{|c|c|c|c|c|c|}
\hline \multirow{2}{*}{$\begin{array}{c}\text { Parâmetros } \\
\text { antropométricos }\end{array}$} & \multicolumn{2}{|c|}{ Classificação do IMC/I } & \multirow{2}{*}{ p-valor ${ }^{a}$} & \multirow{2}{*}{\multicolumn{2}{|c|}{ Kappa/p-valor }} \\
\hline & < adequado & adequado & & & \\
\hline \multicolumn{6}{|l|}{ CB } \\
\hline$<$ adequado & 3 & 6 & \multirow{2}{*}{0,01} & \multirow{2}{*}{0,43} & \multirow{2}{*}{0,001} \\
\hline adequado & 0 & 29 & & & \\
\hline \multicolumn{6}{|l|}{ PCT } \\
\hline$<$ adequado & 1 & 10 & \multirow{2}{*}{0,65} & \multirow{2}{*}{0,02} & \multirow{2}{*}{0,86} \\
\hline adequado & 2 & 25 & & & \\
\hline
\end{tabular}

Nota: ${ }^{\text {a }}$ Teste Exato de Fisher.

Legendas: CB: circunferência do braço; PCT: prega cutânea tricipital; CMB: circunferência muscular do braço.

adequado lhes permite responder melhor ao tratamento oncológico $^{10,13}$.

A desnutrição resulta em menor tolerância ao tratamento, maiores taxas de complicaçóes, recaídas e mortalidade, refletindo em menores taxas de sobrevivência ${ }^{10,13,20}$. Porém, também já vem sendo descrito na literatura que a obesidade pode ser um fator de pior prognóstico ${ }^{21,22}$. Segundo Orgel et al. ${ }^{5}$, pacientes pediátricos com LLA obesos ao diagnóstico apresentaram uma pior resposta à quimioterapia e uma menor sobrevida livre de doença. Esses achados levantam a questão de que esses pacientes que apresentam excesso de peso também devem ser considerados um grupo de risco.

É importante destacar que a prevalência de desnutrição infantil tem mostrado um declínio contínuo, nas últimas quatro décadas, já a prevalência de sobrepeso e obesidade entre crianças e adolescentes brasileiros tem aumentado de forma contínua e expressiva ${ }^{23}$. 
Nos pacientes deste estudo, houve maior frequência de diagnóstico nutricional eutrófico, em todos os parâmetros antropométricos avaliados. Esse achado vai ao encontro de estudos anteriores que também descreveram que os pacientes hematológicos no momento do diagnóstico encontravam-se menos debilitados e com menor comprometimento do estado nutricional quando comparados aos pacientes com tumores sólidos ${ }^{24,25}$. Esse fato pode ser justificado pela patogenia das doenças onco-hematológicas que manifestam de forma aguda os sinais e sintomas, permitindo um diagnóstico de forma precoce.

O estudo de Garófolo et al. ${ }^{4}$ demonstrou que a prevalência da desnutrição variou de acordo com o método utilizado para diagnóstico nutricional. Pacientes com neoplasias hematológicas avaliadas pelo IMC/I apresentavam $6,8 \%$ e pela área muscular do braço $25,4 \%$ de desnutrição, respectivamente. No presente estudo, o IMC/I apresentou $20,4 \%$ e a CMB, 7,9\% de pacientes abaixo do adequado. O IMC/I também foi considerado como abaixo do adequado nos pacientes em risco nutricional e em magreza, talvez por esse motivo apresente valores tão diferentes do estudo de Garófolo et al. ${ }^{4}$. Cabe destacar a importância, na população oncológica com LLA, do critério de risco nutricional pelo IMC/I, pois são pacientes que devem ter seu estado nutricional acompanhado com frequência para traçar uma estratégia de plano nutricional ${ }^{18}$.

Em comparação à avaliação nutricional por diferentes parâmetros antropométricos, não foram encontradas diferenças em 1.154 crianças e adolescentes com câncer sólidos e hematológicos, em um estudo realizado no Brasil $^{26}$. Pode-se observar que a CMB na amostra populacional estudada apresentou associação com o IMC/I, porém a CB e PCT não estavam associadas e tiveram baixa concordância entre elas. Porém, quando avaliadas a CB com a CMB, foi observada uma associação entre as medidas. Segundo alguns estudos ${ }^{1,24}$, a CB é um bom preditor de massa corporal magra, e a PCT é menos precisa como preditor de massa gorda, e subestima esse compartimento da composiçáo corporal em pacientes pediátricos com LLA, assim como o IMC/I.

Em estudo com crianças saudáveis realizado por Bonaccorsi et al. ${ }^{27}$, a CB correlacionou-se fortemente com o IMC. Posteriormente, Barr et al. ${ }^{24}$ demostraram resultado semelhante com amostra de 99 pacientes oncopediátricos; entre os quais, 74 tinham LLA, que foram avaliados ao diagnóstico, e que a $\mathrm{CB}$ possuía uma forte correlação com o IMC. Os resultados do presente estudo corroboram os achados anteriores, sugerindo que a CB é uma medida útil para avaliar o estado nutricional em crianças com câncer, especificamente com LLA, no momento do diagnóstico. Além disso, ela é uma medida fácil e rápida de ser obtida, mesmo em hospitais com poucos recursos, reforçando a importância de realizar essa medida ao diagnóstico e no seguimento nutricional.

Não é de hoje que é descrito que a avaliação nutricional deva ser realizada da forma mais abrangente possível ${ }^{3}$, utilizando não só um parâmetro, mas uma combinação de medidas antropométricas que possam refletir o atual estado nutricional dos pacientes com pediátricos com LLA $^{13,28-30}$. Como o presente estudo foi realizado em uma Instituição de referência em oncologia, a avaliação nutricional completa faz parte da rotina de atendimento nutricional dos pacientes pediátricos. Os achados do presente estudo colaboram para retificar a necessidade de medidas antropométricas simples na avaliação do estado nutricional.

\section{CONCLUSÃO}

Diante do exposto ao diagnóstico na população estudada, o estado nutricional encontra-se dentro do adequado observado pelos parâmetros IMC/I, assim como as demais medidas antropométricas, sendo elas a CB, PCT e CMB. Com esses achados, conclui-se que, ao menos no início do tratamento, as reservas dos compartimentos proteicos e adiposos estáo preservadas. Vale destacar também que, no presente estudo, assim como a associação da CMB com o IMC/I, a CB demonstrou ser um parâmetro sensível para classificar eutrofia.

A avaliação nutricional realizada no momento do diagnóstico é de extrema importância, uma vez que este supostamente seria o momento mais adequado para prevenir o agravamento do estado nutricional, a fim de fornecer uma terapia nutricional mais adequada e individualizada para os pacientes pediátricos com LLA.

\section{CONTRIBUIÇÕES}

Todos os autores contribuíram substancialmente na concepção e no planejamento do estudo; na obtenção, na análise e/ou interpretação dos dados; assim como na redação e revisão crítica e aprovação final da versão publicada.

\section{DECLARAÇÃO DE CONFLITO DE INTERESSES}

Nada a declarar.

\section{FONTES DE FINANCIAMENTO}

Não há. 


\section{REFERÊNCIAS}

1. Barr RD. Nutritional status in children with cancer: before, during and after therapy. Indian J Cancer. 2015;52(2):173-175.

2. Corera Sánchez M, Ariceta Iraola G, Navajas Gutiérrez A, Sasieta Altuna M, Bezanilla Regato JL. Nutritional study of children with oncologic diseases. An Esp Pediatr. 1992;36(4):277-280.

3. Kumar R, Marwaha RK, Bhalla AK, Gulati M. Protein energy malnutrition and skeletal muscle wasting in childhood acute lymphoblastic leukemia. Indian Pediatr. 2000;37(7):720-6.

4. Garófolo A, Lopez FA, Petrilli AS. High prevalence of malnutrition among patients with solid nonhematological tumors as found by using skinfold and circumference measurements. Sáo Paulo Med J. 2005;123(6):277-281.

5. Orgel E, Tucci J, Alhushki W, Malvar J, Sposto R, $\mathrm{Fu} \mathrm{CH}$, et al. Obesity is associated with residual leukemia following induction therapy for childhood B-precursor acute lymphoblastic leukemia. Blood. 2014;124(26):3932-3938.

6. Orgel E, Sposto R, Malvar J, Seibel NL, Ladas E, Gaynon PS, et al. Impact on survival and toxicity by duration of weight extremes during treatment for pediatric acute lymphoblastic leukemia: a report from the children's oncology group. J Clin Oncol. 2014;32(13):1331-1337.

7. Teitelbaum D, Guenter P, Howell WH, Kochevar ME, Roth J, Seidner DL. Definition of terms, style, and conventions used in A.S.P.E.N. guidelines and standards. Nutr Clin Pract. 2005;20(2):281-285.

8. Sociedade Brasileira de Pediatria, Departamento Científico de Nutrologia, editores. Avaliação nutricional da criança e do adolescente: manual de orientação [Internet]. Rio de Janeiro: Sociedade Brasileira de Pediatria; 2009. 112 p. [acesso 2018 Ago 3]. Disponível em: http://www.sbp.com.br/fileadmin/user_upload/ pdfs/MANUAL-AVAL-NUTR2009.pdf

9. Sala A, Pencharz P, Barr RD. Children, cancer, and nutrition-A dynamic triangle in review. Cancer. 2004;100(4):677-687.

10. Loeffen EA, Brinksma A, Miedema KG, de Bock GH, Tissing WJ. Clinical implications of malnutrition in childhood cancer patients--infections and mortality. Supportive Care in Cancer. 2015;23(1):143-150.

11. Garófolo A, Caran EM, Silva NS, Lopez FA. Prevalência de desnutrição em crianças com tumores sólidos. Rev Nutr. 2005;18(2):193-200.

12. Mosby TT, Barr RD, Pencharz PB. Nutritional assessment of children with cancer. J Pediat Oncol Nurs. 2009;26(4):186-197.

13. Sala A, Rossi E, Antillon F, Molina AL, de Maselli T, Bonilla M, et al. Nutritional status at diagnosis is related to clinical outcomes in children and adolescents with cancer: a perspective from Central America. Eur J Cancer. 2012;48(2):243-252.

14. Carvalho AL, Schramm MT, Murad LB, Saraiva DC. Estado Nutricional e Desfechos Clínicos em Pacientes Pediátricos com Leucemia Linfoblástica Aguda. Rev Bras Cancerol. 2016;62(4):329-336.

15. Malta M, Cardoso LO, Bastos FI, Magnanini MM, Silva CM. Iniciativa STROBE: subsídios para a comunicação de estudos observacionais. Rev Saúde Pública. junho de 2010;44(3):559-565.

16. De Onis M, Onyango AW, Borghi E, Siyam A, Nishida C, Siekmanna J. Development of a WHO growth reference for school-aged children and adolescents. Bull World Health Organ. 2007;85(9):660-667.

17. World Health Organization. WHO child growth standards: length/height-for-age, weight-for-age, weightfor-length, weight-for-height and body mass indexfor-age: methods and development [Internet]. Geneva: World Health Organization; c2006 [cited 2018 Ago 3]. Available from: http://www.who.int/childgrowth/ standards/Technical_report.pdf

18. Maldonado-Alcázar A, Núñez-Enríquez JC, García-Ruiz CA, Fajardo-Gutierrez A, Mejía-Arangure JM. Alterations of nutritional status in childhood acute leukemia. In: Mejía-Arangure JM, editor. Clinical epidemiology of acute lymphoblastic leukemia: from the molecules to the clinic [Internet]. United Kingdom: IntechOpen; c2013 [cited 2018 Ago 3]. Chapter 12. Available from: http:// www.intechopen.com/books/clinical-epidemiology-ofacute-lymphoblastic-leukemia-from-the-molecules-tothe-clinic/alterations-of-nutritional-status-in-childhoodacute-leukemia

19. Frisancho AR. New norms of upper limb fat and muscle areas for assessment of nutritional status. Am J Clin Nutr. 1981;34(11):2540-2545.

20. Brinksma A, Roodbol PF, Sulkers E, Hooimeijer HL, Sauer PJ, van Sonderen E, et al. Weight and height in children newly diagnosed with cancer. Pediatr Blood Cancer. 2015;62(2):269-273.

21. Butturini AM, Dorey FJ, Lange BJ, Henry DW, Gaynon PS, Fu C, et al. Obesity and outcome in pediatric acute lymphoblastic leukemia. J Clin Oncol. 2007;25(15):2063-2069.

22. Gelelete CB, Pereira SH, Azevedo AM, Thiago LS, Mundim M, Land MG, et al. Overweight as a prognostic factor in children with acute lymphoblastic leukemia. Obesity. 2011;19(9):1908-1911.

23. Instituto Brasileiro de Geografia e Estatística. Pesquisa de orçamentos familiares 2008-2009: antropometria e estado nutricional de crianças, adolescentes e adultos no Brasil. Rio de Janeiro: IBGE; 2010.

24. Barr R, Collins L, Nayiager T, Doring N, Kennedy C, Halton J, et al. Nutritional status at diagnosis in children 
with cancer. 2.: an assessment by arm anthropometry. J Pediatr Hematol/Oncol. 2011;33(3):e101-e104.

25. Brennan BM, Gill M, Pennells L, Eden OB, Thomas AG, Clayton PE. Insulin-like growth factor I, IGF binding protein 3, and IGFBP protease activity: relation to anthropometric indices in solid tumours or leukaemia. Arch Dis Child. 1999;80(3):226-230.

26. Lemos PDSM, Oliveira FLC, Caran EMM. Nutritional status of children and adolescents at diagnosis of hematological and solid malignancies. Rev Bras Hematol Hemoter. 2014;36(6):420-423.

27. Bonaccorsi G, Baggiani L, Bassetti A, Colombo C, Lorini C, Mantero S, et al. Body composition assessment in a sample of eight-year-old children. Nutrition. 2009;25(10):1020-1028.

28. Abdelhadi RA, Bouma S, Bairdain S, Wolff J, Legro A, Plogsted S, et al. Characteristics of hospitalized children with a diagnosis of malnutrition: United States, 2010. JPEN J Parenter Enteral Nutr. 2016;40(5):623-635.

29. Collins L, Nayiager T, Doring N, Kennedy C, Webber C, Halton J, et al. Nutritional status at diagnosis in children with cancer I. an assessment by dietary recall compared with body mass index and body composition measured by dual energy $\mathrm{x}$-ray absorptiometry. J Pediatr Hematol/ Oncol. 2010;32(8):e299-e303.

30. Kondrup J, Allison SP, Elia M, Vellas B, Plauth M. ESPEN guidelines for nutrition screening 2002. Clinical Nutrition. 2003;22(4):415-421. 